\title{
Stress Relaxation and Targeted Nutrition to Treat Patellar Tendinopathy
}

\author{
Keith Baar \\ University of California Davis
}

\begin{abstract}
Patellar tendinopathy is one of the most common afflictions in jumping sports. This case study outlines the rehabilitation of a professional basketball player diagnosed by magnetic resonance imaging (MRI) with a central core patellar tendinopathy within the proximal enthesis. The player undertook a nutrition and strength-based rehabilitation program combining gelatin ingestion and heavy isometric loading of the patellar tendon designed to produce significant stress relaxation as part of their competition schedule and a whole-body training plan. On follow-up one and a half years into the program an independent orthopedic surgeon declared the tendon normal on MRI. Importantly, the improved MRI results were associated with a decrease in pain and improved performance. This case study provides evidence that a nutritional intervention combined with a rehabilitation program that uses stress relaxation can improve clinical outcomes in elite athletes.
\end{abstract}

Keywords: exercise, jumper's knee, loading, rehabilitation, tendon

Patellar tendinopathy (PT) is an extremely common musculoskeletal issue in elite athletes competing in jumping sports where reports of prevalence reach between $32 \%$ (basketball) and $44.6 \%$ (volleyball), whereas in body weight-supported sports such as cycling, the incidence of PT is low (Lian et al., 2005). Interestingly, subelite athletes report significantly lower PT (14.4\% for volleyball), suggesting that the volume or intensity of loading contributes to the development of this chronic problem (Hagglund et al., 2011; Janssen et al., 2015). PT is especially common in professional basketball due to the high playing load and intensity. In a preliminary report from the Australian National Basketball League, 52.3\% of players reported patellar tendon pain that limited performance (Hannington et al., 2017), suggesting that the prevalence of this injury is high at the elite level.

Typically, severe chronic PT can be visualized by magnetic resonance imaging (MRI) as an increase in signal intensity at the inferior pole of the patella (Johnson et al., 1996), suggesting that it develops from an injury to the proximal enthesis. In nonelite athletes, PT is treated with rest and cryotherapy with limited success (Schwartz et al., 2015). Nonsteroidal anti-inflammatory drugs (NSAIDs) are equivocal as to their effect on tendinopathy (Weiler, 1992). Indeed, recent work suggests that NSAIDs may not even be delivered to human tendons sufficiently to alter gene expression (Heinemeier et al., 2017).

One of the major issues surrounding PT is that stress shielding can occur with loading (Almekinders et al., 2002), specifically at the insertion/origin regions (Orchard et al., 2004). Stress shielding is most commonly associated with the implantation of prosthetics within bone (Sumner, 2015). Essentially, the stronger metal components of the implant shield the neighboring bone from load (stress) resulting in more of the load being transmitted through the metal and subsequent loss of the "unloaded" native bone (Sumner, 2015). By analogy, healthy collagen can shield weaker or injured

Baar is with the Depts. of Neurobiology, Physiology, and Behavior and Physiology and Membrane Biology, University of California Davis, Davis, CA. Address author correspondence to Keith Baar at kbaar@ucdavis.edu. regions within the tendon resulting in unloading and a subsequent decrease in collagen content and orientation within the injured region (Docking \& Cook, 2016). The change in collagen content and orientation within the injured region is further associated with an increase in proteoglycans and water (Samiric et al., 2009), suggesting that the stress-shielded region of the tendon may be placed under a compressive load as the stronger collagen fibers squeeze (compress) the shielded region (Docking et al., 2013; Majima et al., 2000).

One way to overcome stress shielding is to take advantage of stress relaxation within the tendon (Atkinson et al., 1999). Stress relaxation is a fundamental property of viscoelastic tissues like tendons, where force within a tendon decreases over time when a static load is applied and the tendon is held at a constant length (Atkinson et al., 1999). Essentially, this means that when a tendon is loaded using isometric contractions, the collagen fibers/fibrils/ fascicles progressively relax over time, eventually reaching a steady state. As the collagen fibers/fibrils/fascicles relax, stress shielding within the tendon should decrease, and a directional mechanical signal should be imparted to the injured region. Consistent with this hypothesis, isometric loading protocols decrease pain in high-level athletes with tendinopathies (Rio et al., 2015; van Ark et al., 2016); however, whether a consistent program of isometric loading can improve the quality of the injured tendon has yet to be demonstrated.

On top of the positive effects of loading on tendon health, we have recently shown that nutrition can positively affect collagen synthesis in musculoskeletal tissues (Shaw et al., 2017). Briefly, we demonstrated that supplementing with $15 \mathrm{~g}$ of gelatin, $1 \mathrm{~h}$ before loading, resulted in an increase in whole-body collagen synthesis and increased mechanics and collagen content of human engineered ligaments (Shaw et al., 2017). These data suggest that supplementing with gelatin can increase collagen synthesis. Improving collagen synthesis in stress-shielded tendon might be important as immobilization for even short periods (10 days) has been shown to reduce tendon collagen synthesis by up to $50 \%$, and the decline in collagen synthesis continues out to 23 days (de Boer et al., 2007). Even though the decrease in collagen synthesis was 
not associated with a decrease in patellar tendon cross-sectional area, the tendon did show decreased functional performance (de Boer et al., 2007), suggesting that decreased collagen synthesis is associated with impaired tendon function.

This paper presents a case study of a professional basketball player with chronic PT. The player was placed on a program that combined gelatin ingestion and stress relaxation loading as part of a rehabilitation protocol. Of note, even with an extensive playing and training schedule, the protocol significantly decreased MRI reactivity, increased the thickness of the midsubstance of the patellar tendon, and diminished pain. The loading and nutrition protocol presented here therefore could be one component of a rehabilitation protocol to improve functional outcomes in elite athletes.

\section{Player and Imaging}

As part of the NBA scouting combine, all players invited to the event are given an MRI of both knees. For the athlete in question, one of his knees showed significant MRI reactivity at the inferior pole of the patella and MRI reactivity throughout the length of the tendon that was visible both in the sagittal T1-weighted images (Figure 1a) and axial proton density weighted SPectral Attenuated Inversion Recovery images (Figure 2a). At the time, the player was 21 years old and had experienced knee pain for $\sim 5$ years. The initial MRI was completed in May, and the player was drafted that year and began working with the team 3 months later. Follow-up MRIs were performed on a Philips Ingenia 3.0T MR (Philips America,
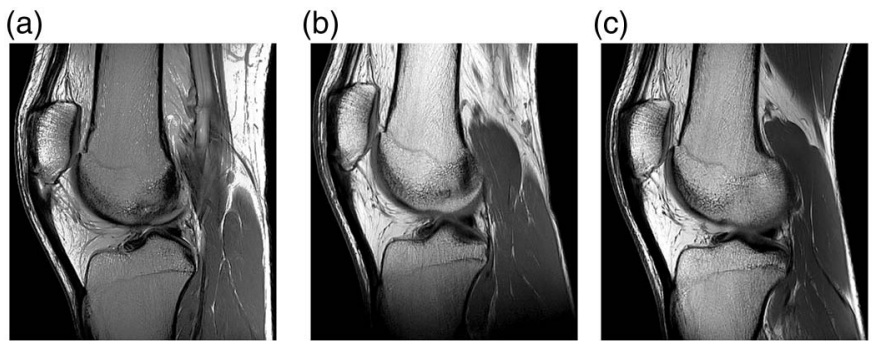

Figure 1 - T1-weighted MRI images from (a) before, (b) 12 months, and (c) 18 months into the intervention showing extent of the patellar tendinopathy within the central core of the inferior pole of the patella (note the white triangular region at the bottom of the patella in panel (a) before the intervention and the progressive regression with the intervention. (a)

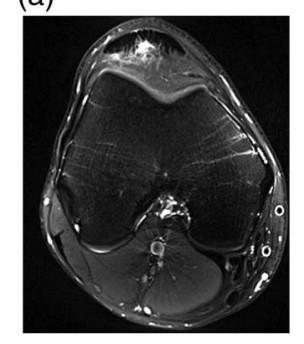

(b)

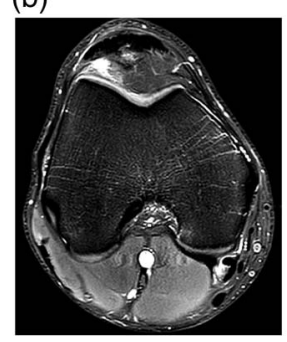

(c)

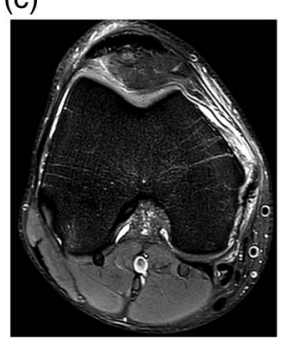

Figure 2 - Axial proton density weighted SPAIR MRI images from (a) before, (b) 12 months, and (c) 18 months into the intervention showing the hole within the proximal patellar tendon before the intervention and the progressive loss of signal at the inferior pole of the patella following the intervention. Note also that the cross-sectional area of the patellar tendon is smaller after rehab. SPAIR indicates SPectral Attenuated Inversion Recovery.
Cambridge, MA) system in August (Figures $1 \mathrm{~b}$ and 2b) of the following year (15 months after the initial scan) and 6 months later (February of his second year in the league; Figures 1c and 2c) to gauge his progression. The initial MRI was completed on a separate machine, but great care was used to validate that equivalent images in the original and follow-up scans were used for comparison. In the sections shown, the same cut was used, and the patellar and tibial areas were similar.

\section{Overview of Interventions}

After being informed of the purpose of the study and of all associated risks, the player gave written consent. The study was performed in accordance with the principles outlined in the Declaration of Helsinki and the University of California Davis Institutional Review Board.

\section{In-Season Loading Volume}

As the player was part of the active team, he participated in more than 50 games per season across two seasons averaging more than 20 min per contest. On top of game loading, he also participated in an average of three organized practices per week as well as a structured strength training program overseen by an elite strength and conditioning coach while being monitored by a rehabilitation specialist, physiotherapist, and sport medicine doctor.

\section{Stress Relaxation Loading}

The patellar tendon targeted training program consisted of midrange isometric holds for two open chain (leg extension and leg press) and one closed chain (Spanish squats) exercise. Both the weight lifted and the duration of the isometric hold progressively increased over the 18 months in the program. The isometric holds started at $10 \mathrm{~s}$ and progressively increased by $5 \mathrm{~s}$ and reached a peak of $30 \mathrm{~s} 6$ months into the program. The stress relaxation program was performed twice a week through preseason, in-season, and offseason workouts. The athlete was asked to perform one to three sets of two to four repetitions of single-leg, midrange isometric holds for leg extension, leg press, or Spanish squats. The holds were performed at $>80 \%$ of one-repetition maximum (1RM), and 1RM was evaluated every other month. The 30-s isometric holds were used because the force in patellar tendon sections drops by $60 \%$ by $30 \mathrm{~s}$ with only a further $10 \%$ decrease by $180 \mathrm{~s}$ (Atkinson et al., $1999)$. The length of the session ( $10 \mathrm{~min}$ from start to finish) was used because we had previously found that short periods of loading (5-10 $\mathrm{min}$ ) were optimal for stimulating the cellular response to load and increasing collagen synthesis (Paxton et al., 2012).

\section{Nutrition Intervention}

Normal dietary intake for the player was not tracked, nor did the player report changes in dietary intake over the length of the study. One hour before the patellar tendon targeted training program, the athlete consumed $15 \mathrm{~g}$ of gelatin with $\sim 225 \mathrm{mg}$ vitamin $\mathrm{C}$. The supplement consisted of commercial baking gelatin (Great Lakes unflavored beef gelatin) in $16 \mathrm{oz}$ of orange juice (containing $\sim 225 \mathrm{mg}$ vitamin $\mathrm{C}$ ). The amount of gelatin was based on our previous work showing that $15 \mathrm{~g}$ of gelatin increased load-dependent collagen synthesis (Shaw et al., 2017). The timing of nutrients prior to exercise was designed to enhance nutrient delivery to the injured tendon and take advantage of cellular processes activated by the exercise stimulus to maximize collagen synthesis. 
Compliance with the loading and nutrition program was greater than $95 \%$ in-season, and because of the early success, the athlete maintained approximately this level of compliance in the off-season.

\section{Body Composition Assessment}

The player underwent body composition assessment via surface anthropometry using the sum of seven skinfolds at regular intervals throughout the program.

\section{Strength Assessment}

Isometric strength was determined using a Cybex 6000 isokinetic dynamometer (Lumex, Inc., Ronkonkoma, NY) calibrated before and after each test according to the manufacturer's instruction manual.

\section{Outcome of the Intervention}

\section{Changes in Body Composition Over the Season (Table 1)}

Over the course of the season, the player saw minimal changes in body mass $(-2.5 \mathrm{~kg})$. The decrease in body weight corresponded with a decrease in fat mass (from $8.4 \%$ to $5.9 \%$ ), indicating that a large percentage of the body mass loss was the result of a decrease in fat mass. In agreement with this assessment, lean mass only changed slightly over the testing period $(75.6 \mathrm{~kg}$ at the time of the initial MRI and $74.7 \mathrm{~kg}$ at the time of the third MRI).

\section{Functional Progression Through the Program}

In spite of a slight decrease in lean mass, maximal single-leg isometric hamstring strength increased $196 \%$, isometric leg extension strength increased by $156 \%$, and leg press strength increased $187.5 \%$ over the period of the program. The stress relaxation sets performed in each session for single-legged isometric leg extensions progressed from three repetitions of $10 \mathrm{~s}$ with $12.5 \mathrm{~kg}$ to two repetitions of $30 \mathrm{~s}$ with $32 \mathrm{~kg}$, whereas the single-legged isometric leg press progressed from three 10 -s holds with $80 \mathrm{~kg}$ to two 30 -s holds with $170 \mathrm{~kg}$.

Table 1 Anthropometric Data Across the Intervention Period

\begin{tabular}{lcccc}
\hline Month & $\begin{array}{c}\text { Height } \\
(\mathbf{c m})\end{array}$ & $\begin{array}{c}\text { Weight } \\
(\mathbf{k g})\end{array}$ & $\begin{array}{c}\text { Skinfold } \\
(\mathbf{m m})\end{array}$ & $\begin{array}{c}\% \\
\text { Body fat }\end{array}$ \\
\hline 0 & 200 & 91.8 & 59.5 & 8.4 \\
3 & 200 & 91.2 & 58.5 & 8.3 \\
5 & 200 & 90.6 & 52 & 7.7 \\
6 & 200 & 89.6 & 41.2 & 6.5 \\
8 & 200 & 88.9 & 40.4 & 6.5 \\
13 & 200 & 91.4 & 39 & 6.3 \\
14 & 200 & 90.5 & 32.6 & 6.3 \\
15 & 200 & 89.5 & 36.4 & 6.7 \\
16 & 200 & 89.6 & 34.4 & 6.4 \\
17 & 200 & 87.1 & 31 & 6.1 \\
18 & 200 & 89.3 & 29.8 & 5.9 \\
\hline
\end{tabular}

\section{Imaging and Pain}

Follow-up MRI images were performed after a year in the program (15 months after the first MRI) and a further 6 months later, after the player had been on the program for $\sim 18$ months. The MRI images following 12 and 18 months in the program showed a progressive decrease in MRI reactivity at the inferior pole of the patella in both the sagittal T1-weighted images (Figure 1) and the axial SPectral Attenuated Inversion Recovery images (Figure 2). At the time of the third scan, a blinded orthopedic surgeon declared the patellar tendon normal. The reduction of reactivity at the inferior pole of the patella was accompanied by a decrease in the diameter of the patellar tendon at the proximal enthesis from $10.8 \mathrm{~mm}$ at Scan 1 to 8.7 and $8.16 \mathrm{~mm}$ in Scans 2 and 3, respectively. The decrease in the thickness of the proximal end of the patellar tendon was accompanied by an increase in the thickness at the midpoint of the tendon. The diameter of the midpoint of the patellar tendon progressively increased from $5.74 \mathrm{~mm}$ (Scan 1) to $6.16 \mathrm{~mm}$ (Scan 2), and then $6.29 \mathrm{~mm}$ (Scan 3). This was accompanied by a decrease in $\mathrm{T} 1$ and $\mathrm{T} 2$ reactivity (not shown). Therefore, the proximal end of the tendon decreased $\sim 25 \%$, whereas the middle of the tendon hypertrophied $\sim 10 \%$. Beyond the changes in imaging, self-reported pain and tenderness within the tendon decreased. At the point of the final scan, the athlete was pain free, even though he was playing more than $25 \mathrm{~min}$ a game.

\section{Reflections}

The goal of this case study was to determine whether a targeted loading and nutritional program could enhance the outcomes of a PT rehabilitation program. Specifically, we used a loading program that maximized stress relaxation (long isometric holds) in the hopes of providing a uniaxial tensile load to the injured region of the patellar tendon. Together with the stress relaxation loading, we primed the athlete with $15 \mathrm{~g}$ of gelatin containing $225 \mathrm{mg}$ of vitamin $\mathrm{C}$, so that once the cells were activated by the load, they would have the substrates necessary to increase collagen synthesis. The major results of the program were a decrease in pain and tenderness that corresponded with a significant decrease in MRI reactivity at the inferior pole of the patella and an increase in midsubstance patellar tendon thickness.

Stress relaxation is a fundamental property of viscoelastic materials like tendons and ligaments (Kjaer, 2004). Basically, as the tissue is loaded water is squeezed out and structures, such as fascicles and subfascicular components of the tendon slide relative to each other resulting in a progressive decrease in the tension within the tendon at a given length (Atkinson et al., 1999; Magnusson et al., 1997). In the human patellar tendon, two important observations about stress relaxation were first made by Atkinson et al. (1999). First, stress relaxation is bigger when larger parts of a tendon are loaded. This is important as it suggests that intrinsic differences in the strength of different fascicle or subfascicle regions are exposed during stress relaxation. In the current case, this is potentially important as it suggests that stronger regions of the tendon may relax early in the process and weaker/ damaged regions of the tendon may be forced to take on more of the load as stress relaxation progresses. As directional loading is fundamentally required for high degrees of collagen synthesis and matrix alignment (Kapacee et al., 2008), this suggests that stress relaxation can be used to provide a tensile stimulus to damaged regions of the collagen matrix to promote directional collagen synthesis. The second important observation by Atkinson et al. (1999) was that stress relaxation reaches $\sim 60 \%$ within $30 \mathrm{~s}$ and 
continues only a further $10 \%$ more up to $180 \mathrm{~s}$. This suggests that heavy isometric holds lasting $30 \mathrm{~s}$ will be sufficient to provide a similar degree of stress relaxation as holds lasting six times longer. Therefore, the rationale for the loading paradigm was to use stress relaxation to provide a tensile load to the cells within the damaged central core of the proximal patellar tendon.

As important as tensile loading is to collagen synthesis, recent work from our group (Shaw et al., 2017) and others (Clark et al., 2008; McAlindon et al., 2011; Oesser et al., 1999) suggests that collagen synthesis can be enhanced by supplementing the diet with gelatin or hydrolyzed collagen. Specifically, we have previously shown that providing $15 \mathrm{~g}$ of gelatin together with $\sim 50 \mathrm{mg}$ of vitamin $\mathrm{C}$ an hour before loading resulted in an increase in collagen synthesis relative to a placebo or 5-g dose of gelatin (Shaw et al., 2017). Therefore, for this case study, we had the athlete consume $15 \mathrm{~g}$ of gelatin within an orange juice slurry (containing $225 \mathrm{mg}$ of vitamin C) $1 \mathrm{~h}$ before the stress relaxation training. Even though we do not have a control group for this intervention, we have growing evidence that specific amino acids within the gelatin provide a stimulus to the cells of a tendon or ligament to increase collagen synthesis. Coupled with the directional tensile load, the added amino acids may be necessary to support the increase in collagen synthesis. However, much more work is needed before we can definitively understand the role of gelatin in the recovery described in this case study.

As with any intervention with elite athletes, the intervention can only be effective if there is buy in from the player. In this case, the injury was sufficiently painful that the player was willing to drink the slurry and perform the loading paradigm twice a week. When the treatment showed success, the player quickly became a major advocate of the program within the team. Currently, any player with symptoms of patellar or other forms of tendinopathy quickly opts into the program, and now most members of the team preform a form of prehab using the gelatin and stress relaxation loading along with their regular training. Even though we have not quantified musculoskeletal injuries before and after the intervention, reports suggest that visits to the training room and noncontact musculoskeletal injuries within the team have dropped.

Tendinopathies are multifactorial in nature, and central core tendinopathies are particularly difficult to prevent and treat in an elite population. We hope that the program reported here can provide a starting place for treating these debilitating injuries.

\section{Acknowledgments}

The author wishes to thank the athlete who undertook this rehabilitation protocol and followed instructions diligently. In addition, this project would not have been possible without the expertise, experience, and willingness of the Director of Strength and Conditioning and his staff, the Head Athletic Trainer and their staff, and the Director of Player Health and Performance. The author is indebted to them for their efforts and willingness to publish the results. Finally, thanks are owed to Dr. Letitia Bradford for independent evaluation of the blinded MRI images. The author has no conflicts of interest in regards to this case study. The results of the study are presented clearly, honestly, and without fabrication, falsification, or inappropriate data manipulation.

\section{References}

Almekinders, L.C., Vellema, J.H., \& Weinhold, P.S. (2002). Strain patterns in the patellar tendon and the implications for patellar tendinopathy. Knee Surgery, Sports Traumatology, Arthroscopy, 10(1), 2-5. PubMed ID: 11819013 doi:10.1007/s001670100224

Atkinson, T.S., Ewers, B.J., \& Haut, R.C. (1999). The tensile and stress relaxation responses of human patellar tendon varies with specimen cross-sectional area. Journal of Biomechanics, 32(9), 907-914. PubMed ID: 10460127 doi:10.1016/S0021-9290(99)00089-5

Clark, K.L., Sebastianelli, W., Flechsenhar, K.R., Aukermann, D.F., Meza, F., Millard, R.L., . . A Albert, A. (2008). 24-Week study on the use of collagen hydrolysate as a dietary supplement in athletes with activity-related joint pain. Current Medical Research and Opinion, 24(5), 1485-1496. PubMed ID: 18416885 doi:10.1185/ 030079908 X291967

de Boer, M.D., Maganaris, C.N., Seynnes, O.R., Rennie, M.J., \& Narici, M.V. (2007). Time course of muscular, neural and tendinous adaptations to 23 day unilateral lower-limb suspension in young men. The Journal of Physiology, 583(Pt 3), 1079-1091. PubMed ID: 17656438 doi:10.1113/jphysiol.2007.135392

Docking, S., Samiric, T., Scase, E., Purdam, C., \& Cook, J. (2013). Relationship between compressive loading and ECM changes in tendons. Muscle, Ligaments and Tendons Journal, 3(1), 7-11. PubMed ID: 23885340 doi:10.11138/mltj/2013.3.1.007

Docking, S.I., \& Cook, J. (2016). Pathological tendons maintain sufficient aligned fibrillar structure on ultrasound tissue characterization (UTC). Scandinavian Journal of Medicine \& Science in Sports, 26(6), 675-683. PubMed ID: 26059532 doi:10.1111/sms.12491

Hagglund, M., Zwerver, J., \& Ekstrand, J. (2011). Epidemiology of patellar tendinopathy in elite male soccer players. American Journal of Sports Medicine, 39(9), 1906-1911. PubMed ID: 21642599 doi:10. 1177/0363546511408877

Hannington, M., Rio, E., Padua, D., Stanley, L., Berkoff, D., Edwards, S., . . . Docking, S. (2017). Prevalence and impact of patellar tendinopathy on elite basketball athletes: Quantifying injury beyond the time-loss definition. Journal of Science and Medicine in Sport, 20(S3), 17-18. doi:10.1016/j.jsams.2017.09.223

Heinemeier, K.M., Ohlenschlaeger, T.F., Mikkelsen, U.R., Sonder, F., Schjerling, P., Svensson, R.B., \& Kjaer, M. (2017). Effects of antiinflammatory (NSAID) treatment on human tendinopathic tissue. Journal of Applied Physiology, 123(5), 1397-1405. PubMed ID: 28860166 doi:10.1152/japplphysiol.00281.2017

Janssen, I., Steele, J.R., Munro, B.J., \& Brown, N.A. (2015). Previously identified patellar tendinopathy risk factors differ between elite and sub-elite volleyball players. Scandinavian Journal of Medicine \& Science in Sports, 25(3), 308-314. PubMed ID: 25983242 doi:10. 1111/sms.12206

Johnson, D.P., Wakeley, C.J., \& Watt, I. (1996). Magnetic resonance imaging of patellar tendonitis. The Journal of Bone and Joint Surgery. British Volume, 78(3), 452-457. PubMed ID: 8636185 doi:10.1302/ 0301-620X.78B3.0780452

Kapacee, Z., Richardson, S.H., Lu, Y., Starborg, T., Holmes, D.F., Baar, K., \& Kadler, K.E. (2008). Tension is required for fibripositor formation. Matrix Biology, 27(4), 371-375. PubMed ID: 18262777 doi:10.1016/j.matbio.2007.11.006

Kjaer, M. (2004). Role of extracellular matrix in adaptation of tendon and skeletal muscle to mechanical loading. Physiological Reviews, 84(2), 649-698. PubMed ID: 15044685 doi:10.1152/physrev. 00031.2003

Lian, O.B., Engebretsen, L., \& Bahr, R. (2005). Prevalence of jumper's knee among elite athletes from different sports: A cross-sectional study. The American Journal of Sports Medicine, 33(4), 561-567. PubMed ID: 15722279 doi:10.1177/0363546504270454

Magnusson, S.P., Simonsen, E.B., Aagaard, P., Boesen, J., Johannsen, F., \& Kjaer, M. (1997). Determinants of musculoskeletal flexibility: 
Viscoelastic properties, cross-sectional area, EMG and stretch tolerance. Scandinavian Journal of Medicine \& Science in Sports, 7(4), 195-202. PubMed ID: 9241023 doi:10.1111/j.1600-0838.1997. tb00139.x

Majima, T., Marchuk, L.L., Sciore, P., Shrive, N.G., Frank, C.B., \& Hart, D.A. (2000). Compressive compared with tensile loading of medial collateral ligament scar in vitro uniquely influences mRNA levels for aggrecan, collagen type II, and collagenase. Journal of Orthopaedic Research, 18(4), 524-531. PubMed ID: 11052487 doi:10.1002/jor. 1100180403

McAlindon, T.E., Nuite, M., Krishnan, N., Ruthazer, R., Price, L.L., Burstein, D., .. . Flechsenhar, K. (2011). Change in knee osteoarthritis cartilage detected by delayed gadolinium enhanced magnetic resonance imaging following treatment with collagen hydrolysate: A pilot randomized controlled trial. Osteoarthritis and Cartilage, 19(4), 399-405. PubMed ID: 21251991 doi:10.1016/j.joca.2011.01.001

Oesser, S., Adam, M., Babel, W., \& Seifert, J. (1999). Oral administration of ${ }^{14} \mathrm{C}$ labeled gelatin hydrolysate leads to an accumulation of radioactivity in cartilage of mice (C57/BL). The Journal of Nutrition, 129(10), 1891-1895. PubMed ID: 10498764 doi:10.1093/jn/129.10.1891

Orchard, J.W., Cook, J.L., \& Halpin, N. (2004). Stress-shielding as a cause of insertional tendinopathy: The operative technique of limited adductor tenotomy supports this theory. Journal of Science and Medicine in Sport, 7(4), 424-428. PubMed ID: 15712497 doi:10. 1016/S1440-2440(04)80259-7

Paxton, J.Z., Hagerty, P., Andrick, J.J., \& Baar, K. (2012). Optimizing an intermittent stretch paradigm using ERK1/2 phosphorylation results in increased collagen synthesis in engineered ligaments. Tissue Engineering. Part A, 18(3-4), 277-284. PubMed ID: 21902469 doi:10.1089/ten.tea.2011.0336
Rio, E., Kidgell, D., Purdam, C., Gaida, J., Moseley, G.L., Pearce, A.J., \& Cook, J. (2015). Isometric exercise induces analgesia and reduces inhibition in patellar tendinopathy. British Journal of Sports Medicine, 49(19), 1277-1283. PubMed ID: 25979840 doi:10.1136/ bjsports-2014-094386

Samiric, T., Parkinson, J., Ilic, M.Z., Cook, J., Feller, J.A., \& Handley, C.J. (2009). Changes in the composition of the extracellular matrix in patellar tendinopathy. Matrix Biology, 28(4), 230-236. PubMed ID: 19371780 doi:10.1016/j.matbio.2009.04.001

Schwartz, A., Watson, J.N., \& Hutchinson, M.R. (2015). Patellar tendinopathy. Sports Health, 7(5), 415-420. PubMed ID: 26502416 doi:10. $1177 / 1941738114568775$

Shaw, G., Lee-Barthel, A., Ross, M.L., Wang, B., \& Baar, K. (2017). Vitamin C-enriched gelatin supplementation before intermittent activity augments collagen synthesis. The American Journal of Clinical Nutrition, 105(1), 136-143. PubMed ID: 27852613 doi:10.3945/ajen.116.138594

Sumner, D.R. (2015). Long-term implant fixation and stress-shielding in total hip replacement. Journal of Biomechanics, 48(5), 797-800. PubMed ID: 25579990 doi:10.1016/j.jbiomech.2014.12.021

van Ark, M., Cook, J.L., Docking, S.I., Zwerver, J., Gaida, J.E., van den Akker-Scheek, I., \& Rio, E. (2016). Do isometric and isotonic exercise programs reduce pain in athletes with patellar tendinopathy in-season? A randomised clinical trial. Journal of Science and Medicine in Sport, 19(9), 702-706. PubMed ID: 26707957 doi:10. 1016/j.jsams.2015.11.006

Weiler, J.M. (1992). Medical modifiers of sports injury. The use of nonsteroidal anti-inflammatory drugs (NSAIDs) in sports soft-tissue injury. Clinics in Sports Medicine, 11(3), 625-644. PubMed ID: 1638643 